\title{
STABILITY OF THE REVERSE BLASCHKE-SANTALÓ INEQUALITY FOR UNCONDITIONAL CONVEX BODIES
}

\author{
JAEGIL KIM AND ARTEM ZVAVITCH \\ (Communicated by Thomas Schlumprecht)
}

\begin{abstract}
Mahler's conjecture asks whether the cube is a minimizer for the volume product of a body and its polar in the class of symmetric convex bodies in $\mathbb{R}^{n}$. The inequality corresponding to the conjecture is sometimes called the reverse Blaschke-Santaló inequality. The conjecture is known to be true in $\mathbb{R}^{2}$ and for several special cases. In the class of unconditional convex bodies, Saint-Raymond confirmed the conjecture, and Meyer and Reisner, independently, characterized the equality case. In this paper we present a stability version of these results and also show that any symmetric convex body, which is sufficiently close to an unconditional body, satisfies the reverse BlaschkeSantaló inequality.
\end{abstract}

\section{INTRODUCTION}

As usual, we denote by $\langle x, y\rangle$ the inner product of two vectors $x, y \in \mathbb{R}^{n}$ and by $|x|$ the length of a vector $x \in \mathbb{R}^{n}$. A convex body is a compact convex subset of $\mathbb{R}^{n}$ with non-empty interior. We say that a set $K$ is symmetric if it is centrally symmetric with center at the origin, i.e. for every $x \in K$ we get $-x \in K$. A set $K \subset \mathbb{R}^{n}$ is said to be unconditional if it is symmetric with respect to any coordinate hyperplane, i.e., $\left( \pm x_{1}, \pm x_{2}, \ldots, \pm x_{n}\right) \in K$, for any $x \in K$ and any choice of \pm signs.

We write $|A|$ for the $k$-dimensional Lebesgue measure (volume) of a measurable set $A \subset \mathbb{R}^{n}$, where $k=1, \ldots, n$ is the dimension of the minimal flat containing $A$. The polar body $K^{\circ}$ of a symmetric convex body $K$ is defined by

$$
K^{\circ}=\left\{y \in \mathbb{R}^{n} \mid\langle x, y\rangle \leq 1 \text { for all } x \in K\right\} .
$$

The volume product of a symmetric convex body $K$ is defined by

$$
\mathcal{P}(K)=|K|\left|K^{\circ}\right|
$$

We note that the notion of the volume product (as well as polarity) can be generalized to the non-symmetric setting (see for example [42, p. 419]), but in this paper we will focus on the questions related to the centrally symmetric bodies. It turns out that the volume product is invariant under the polarity and under invertible

\footnotetext{
Received by the editors March 2, 2013 and, in revised form, August 12, 2013 and August 15, 2013.

2010 Mathematics Subject Classification. Primary 52A20, 53A15, 52B10.

Key words and phrases. Convex bodies, polar bodies, unconditional convex bodies, Hanner polytopes, volume product, Mahler's conjecture, Blaschke-Santaló inequality.

The authors were supported in part by U.S. National Science Foundation grant DMS-1101636, and the first author is also supported in part by NSERC.
} 
linear transformations on $\mathbb{R}^{n}$, that is, for any $T \in \mathrm{GL}(n)$,

$$
\mathcal{P}(T K)=\mathcal{P}(K) \quad \text { and } \quad \mathcal{P}\left(K^{\circ}\right)=\mathcal{P}(K) .
$$

The above property makes the Banach-Mazur distance between symmetric convex bodies $K$ and $L$

$$
\mathrm{d}_{\mathcal{B M}}(K, L)=\inf \{d \geq 1: L \subset T K \subset d L, \text { for some } T \in \operatorname{GL}(n)\},
$$

extremely useful in studying the properties of the volume product. For example, F. John's theorem [19] and the continuity of the volume function with respect to the Banach-Mazur distance guarantee that the volume product attains its maximum and minimum. The maximum for the volume product is provided by the BlaschkeSantaló inequality:

$$
\mathcal{P}(K) \leq \mathcal{P}\left(B_{2}^{n}\right), \text { for all symmetric convex bodies } K \subset \mathbb{R}^{n},
$$

where $B_{2}^{n}$ is the Euclidean unit ball and equality in the above inequality holds only for ellipsoids [29, 34, 40]. Recently, the stability versions of the Blaschke-Santaló inequality were studied in [3,5].

For the minimum of the volume product, it was conjectured by Mahler in [24,25] that $\mathcal{P}(K)$ is minimized at the cube in the class of symmetric convex bodies in $\mathbb{R}^{n}$. In other words, the conjecture asks whether the following inequality is true:

$$
\mathcal{P}(K) \geq \mathcal{P}\left(B_{\infty}^{n}\right), \text { for all symmetric convex bodies } K \subset \mathbb{R}^{n},
$$

where $B_{\infty}^{n}$ is the unit cube. The case of $n=2$ was proved by Mahler [24]. It was also proved in several special cases, like, e.g., unconditional bodies [27, 36, 43, convex bodies having hyperplane symmetries which fix only one common point [4], zonoids 6, 16, 35, bodies of revolution [30, and bodies with some positive curvature assumption [15, 38, 44. An isomorphic version of the conjectures was proved by Bourgain and Milman [8]: there is a universal constant $c>0$ such that $\mathcal{P}(K) \geq c^{n} \mathcal{P}\left(B_{2}^{n}\right)$; see also different proofs in [14,23,32. Functional versions of Blaschke-Santaló inequality and Mahler's conjecture in terms of log-concave functions were investigated by Ball [2, Artstein, Klartag, Milman [1, Fradelizi, Meyer [10 12. The case of equality for unconditional log-concave functions was established by Fradelizi, Gordon, Meyer and Reisner 9]. For more information on Mahler's conjecture, see expository articles [26, 39, 45].

The local minimality of the volume product was first studied in 33 by proving that the cube is a strict local minimizer of the volume product in the class of symmetric convex bodies endowed with the Banach-Mazur distance (see 22 for the local minimality at the simplex in the non-symmetric setting). The result was used by Böröczky and Hug [6] to provide a stability version of (3) for zonoids, namely, a zonoid $Z$ is close in the Banach-Mazur distance to the cube whenever $\mathcal{P}(Z)$ is close to $\mathcal{P}\left(B_{\infty}^{n}\right)$.

The main goal of this paper is to provide a stability version of (3) for unconditional convex bodies. Before stating the theorem we need to recall the definition of a Hanner polytope 17, 18: a symmetric convex body $H$ is called a Hanner polytope if $H$ is one-dimensional, or it is the $\ell_{1}$ or $\ell_{\infty}$ sum of two (lower dimensional) Hanner polytopes. It can be calculated (see for example [39]) that the volume product of the cube is the same as that of Hanner polytopes. Thus every Hanner polytope is also a candidate for being a minimizer of the volume product among symmetric 
convex bodies. It was also shown in [27, 36] that Hanner polytopes are the only possible minimizers in the class of unconditional bodies.

In Section 2 we will prove that if the volume product of an unconditional convex body is sufficiently close to that of the cube, then the body must be close to a Hanner polytope:

Theorem 1. Let $K$ be an unconditional convex body in $\mathbb{R}^{n}$. If

$$
\left|\mathcal{P}(K)-\mathcal{P}\left(B_{\infty}^{n}\right)\right| \leq \varepsilon
$$

for $0<\varepsilon \leq \varepsilon(n)$, then there exists a Hanner polytope $H \subset \mathbb{R}^{n}$ such that

$$
\mathrm{d}_{\mathcal{B M}}(K, H) \leq 1+c(n) \varepsilon,
$$

where $\varepsilon(n), c(n)>0$ are constants depending on $n$ only.

We would like to note that the $\mathbb{R}^{2}$-case of Theorem 1 was proved as a part of more general stability result on $\mathbb{R}^{2}$ by Böröczky, Makai, Meyer and Reisner in [7], so in this paper we will mainly concentrate on the case $n \geq 3$.

Recently it was proved in [20] that a Hanner polytope is a local minimizer of the volume product in the symmetric setting (see the exact statement of the theorem in the beginning of Section 3). In Section 3 we will use this fact and Theorem 1 to prove that any symmetric convex body, which is sufficiently close to an unconditional body, satisfies (3):

Theorem 2. Let $K$ be a symmetric convex body in $\mathbb{R}^{n}$ with

$$
\min \left\{\mathrm{d}_{\mathcal{B M}}(K, L): L \subset \mathbb{R}^{n} \text { unconditional convex body }\right\}=1+\varepsilon,
$$

for $0<\varepsilon \leq \varepsilon(n)$. Then,

$$
\mathcal{P}(K) \geq(1+c(n) \varepsilon) \cdot \mathcal{P}\left(B_{\infty}^{n}\right),
$$

where $\varepsilon(n), c(n)>0$ are constants depending on dimension $n$ only.

\section{Stability of the ReVerse Blaschke-Santaló inequality for UNCONDITIONAL CONVEX BODIES}

Let $e_{1}, \ldots e_{n}$ be the standard orthonormal basis of $\mathbb{R}^{n}$. Denote by $\theta^{\perp}$ the hyperplane orthogonal to a unit vector $\theta$, and by $K \cap \theta^{\perp}, K \mid \theta^{\perp}$ the section of $K$ by $\theta^{\perp}$, the orthogonal projection of $K$ to $\theta^{\perp}$, respectively. Let $\mathbb{R}_{+}^{n}=\left\{x \in \mathbb{R}^{n}\right.$ : $\left.x_{i} \geq 0, \forall i=1, \ldots, n\right\}$ and $K^{+}=K \cap \mathbb{R}_{+}^{n}$. To prove Theorem 1 we first prove the following lemma which is based on the inductive argument of Meyer (see [27] or [39]):

Lemma 1. Consider $\varepsilon \geq 0$ and an unconditional convex body $K \subset \mathbb{R}^{n}, n \geq 2$ such that

$$
\mathcal{P}(K) \leq(1+\varepsilon) \mathcal{P}\left(B_{\infty}^{n}\right)
$$

Then

$$
\mathcal{P}\left(K \cap e_{j}^{\perp}\right) \leq(1+n \varepsilon) \mathcal{P}\left(B_{\infty}^{n-1}\right), \quad j=1, \ldots, n .
$$

Proof. Consider $x \in K^{+}$and $n$ pyramids created by taking the convex hull of $x$ and the intersection of $K^{+}$with each coordinate hyperplane. More precisely, let 
$K_{i}^{+}=\operatorname{conv}\left\{x, K^{+} \cap e_{i}^{\perp}\right\}$ for $i=1, \ldots, n$. Then, using symmetry of $K$ with respect to coordinate hyperplanes,

$$
|K|=2^{n}\left|K^{+}\right| \geq 2^{n}\left|\bigcup_{i=1}^{n} K_{i}^{+}\right|=2^{n} \sum_{i=1}^{n} \frac{1}{n}\left\langle x, e_{i}\right\rangle \frac{\left|K \cap e_{i}^{\perp}\right|}{2^{n-1}}=\sum_{i=1}^{n}\left\langle x, e_{i}\right\rangle \cdot \frac{2\left|K \cap e_{i}^{\perp}\right|}{n},
$$

or equivalently,

$$
\left\langle x, \sum_{i=1}^{n} \frac{2\left|K \cap e_{i}^{\perp}\right|}{n|K|} e_{i}\right\rangle \leq 1, \text { for all } x \in K^{+} .
$$

Note that, by the unconditionality of $K$, the above inequality holds for all $x \in K$, so

$$
\sum_{i=1}^{n} \frac{2\left|K \cap e_{i}^{\perp}\right|}{n|K|} e_{i} \in K^{\circ}
$$

Applying the same argument to $K^{\circ}$ we get

$$
\sum_{i=1}^{n} \frac{2\left|K^{\circ} \cap e_{i}^{\perp}\right|}{n\left|K^{\circ}\right|} e_{i} \in K
$$

Thus, using the definition of polarity we get

$$
\sum_{i=1}^{n} \frac{2\left|K \cap e_{i}^{\perp}\right|}{n|K|} \times \frac{2\left|K^{\circ} \cap e_{i}^{\perp}\right|}{n\left|K^{\circ}\right|} \leq 1
$$

Next notice that

$$
K^{\circ} \cap e_{i}^{\perp}=\left(K \mid e_{i}^{\perp}\right)^{\circ}=\left(K \cap e_{i}^{\perp}\right)^{\circ},
$$

where the last equality follows from the unconditionality of $K$. Finally from (5), (6) we get

$$
\mathcal{P}(K) \geq \frac{4}{n^{2}} \sum_{i=1}^{n}\left|K \cap e_{i}^{\perp}\right| \times\left|\left(K \cap e_{i}^{\perp}\right)^{\circ}\right|=\frac{4}{n^{2}} \sum_{j=1}^{n} \mathcal{P}\left(K \cap e_{j}^{\perp}\right) .
$$

Now, we will use (7) to prove our lemma. Since $\mathcal{P}(K) \leq(1+\varepsilon) \mathcal{P}\left(B_{\infty}^{n}\right)$ and $\mathcal{P}\left(B_{\infty}^{n}\right)=\frac{4}{n^{2}} \sum_{j=1}^{n} \mathcal{P}\left(B_{\infty}^{n-1}\right)$, we get

$$
\frac{4}{n^{2}} \sum_{j=1}^{n}(1+\varepsilon) \mathcal{P}\left(B_{\infty}^{n-1}\right)=(1+\varepsilon) \mathcal{P}\left(B_{\infty}^{n}\right) \geq \mathcal{P}(K) \geq \frac{4}{n^{2}} \sum_{j=1}^{n} \mathcal{P}\left(K \cap e_{j}^{\perp}\right) .
$$

It implies that

$$
\mathcal{P}\left(K \cap e_{j}^{\perp}\right) \leq(1+n \varepsilon) \mathcal{P}\left(B_{\infty}^{n-1}\right), \quad j=1, \ldots, n .
$$

Indeed, if $\mathcal{P}\left(K \cap e_{1}^{\perp}\right)>(1+n \varepsilon) \mathcal{P}\left(B_{\infty}^{n-1}\right)$, then

(8) $\sum_{j=1}^{n} \mathcal{P}\left(K \cap e_{j}^{\perp}\right)>(1+n \varepsilon) \mathcal{P}\left(B_{\infty}^{n-1}\right)+(n-1) \mathcal{P}\left(B_{\infty}^{n-1}\right)=\frac{n^{2}}{4}(1+\varepsilon) \mathcal{P}\left(B_{\infty}^{n}\right)$,

where in the first inequality we used our assumption for the $e_{1}^{\perp}$-section and the reverse Blaschke-Santaló inequality (3) for unconditional convex bodies $K \cap e_{i}^{\perp}$, $i=2, \ldots, n$. Finally note that (8) together with (7) gives $\mathcal{P}(K)>(1+\varepsilon) \mathcal{P}\left(B_{\infty}^{n}\right)$; a contradiction!

The next lemma will help us to treat the case of a convex body $K \subset \mathbb{R}^{n}$ whose sections by coordinate hyperplanes are close to the $(n-1)$-dimensional cube. 
Lemma 2. Let $K \subset B_{\infty}^{n}$ be a convex body in $\mathbb{R}^{n}, n \geq 3$, satisfying

$$
K \cap e_{j}^{\perp}=B_{\infty}^{n} \cap e_{j}^{\perp}, \quad \forall j=1, \ldots, n .
$$

Let $p=(t, \ldots, t) \in \partial K \cap \mathbb{R}_{+}^{n}$. Then

$$
|K|\left|K^{\circ}\right| \geq(1+c(1-t))\left|B_{1}^{n}\right|\left|B_{\infty}^{n}\right|
$$

where $c=c(n)>0$ is a constant depending on $n$ only.

Remark. The proof of Lemma2 does not require the assumption of unconditionality of $K$. Such assumption would make the proof a bit shorter and would improve the constant $c(n)$.

Proof. Using (9) we claim that $K$ contains $n$ vectors of the form $(1, \ldots, 1,0,1, \ldots, 1)$ (i.e. $n-1$ coordinates are equal to 1 and one coordinate is equal to 0 ). Moreover, $K$ must contain the convex hull of those points. Thus $\left\{x:\left\langle x, e_{1}+\cdots+e_{n}\right\rangle=\right.$ $n-1\} \cap B_{\infty}^{n} \subset K$, which gives $t \geq(n-1) / n$. Next we choose $q \in \partial K^{\circ}$ such that $\langle p, q\rangle=1$. Here, (9) guarantees that the normal vector to $\partial K$ at $p$ belongs to $\mathbb{R}_{+}^{n}$, and thus we may assume $q \in \mathbb{R}_{+}^{n}$. Let

$$
\begin{aligned}
& P=\operatorname{conv}\left(\{p\} \cup\left\{x \in B_{\infty}^{n} \cap \mathbb{R}_{+}^{n}:\left\langle x, e_{1}+\cdots+e_{n}\right\rangle \leq n-1\right\}\right) \\
& Q=\operatorname{conv}\left(\{q\} \cup B_{1}^{n} \cap \mathbb{R}_{+}^{n}\right) .
\end{aligned}
$$

Then $K \cap \mathbb{R}_{+}^{n} \supset P$. Also from $K \subset B_{\infty}^{n}$ we get $K^{\circ} \supset B_{1}^{n}$ and thus $K^{\circ} \cap$ $\mathbb{R}_{+}^{n} \supset Q$. Next we notice that $q$ belongs to the hyperplane with normal vector $(1 / \sqrt{n}, \ldots, 1 / \sqrt{n})$ whose distance from the origin is $1 /(t \sqrt{n})$. Thus

$$
\begin{aligned}
& |P|=1-\frac{1}{n !}+\frac{1}{n !} \cdot \frac{t \sqrt{n}-\frac{n-1}{n} \sqrt{n}}{1 / \sqrt{n}}=1-\frac{1-t}{(n-1) !}, \\
& |Q|=\frac{1}{n !} \cdot \frac{1 /(\sqrt{n} t)}{1 / \sqrt{n}}=\frac{1}{n ! t} .
\end{aligned}
$$

It gives

$$
|P||Q|=\frac{1}{n !}\left(1+\left[1-\frac{1}{(n-1) !}\right] \frac{1-t}{t}\right) \geq 4^{-n}\left|B_{\infty}^{n}\right|\left|B_{1}^{n}\right|\left(1+c_{n}(1-t)\right),
$$

where $c_{n}=1-\frac{1}{(n-1) !}$ is a positive constant in case of $n \geq 3$. Note also that we have $|P||Q| \geq 4^{-n}\left|B_{\infty}^{n}\right|\left|B_{1}^{n}\right|$, independently of the position of $p$ (i.e. independently of the lower/upper bounds on $t$ ).

If we would assume that $K$ is unconditional then we would be able to finish the proof by simply multiplying (10) by $4^{n}$. In all other cases we must split $K$ and $K^{\circ}$ into $4^{n}$ parts each depending on the choice of signs of coordinates. Construct $P$ and $Q$ corresponding to each part, compute the corresponding volumes, and take the sum. We may apply (10) to the part corresponding to $\mathbb{R}_{+}^{n}$. In all other parts we do not care about the exact position of the point on the boundary, so we can 
estimate the volume using the remark after (10). More precisely,

$$
|K|\left|K^{\circ}\right|=\left(\sum_{\delta}\left|K_{\delta}\right|\right)\left(\sum_{\delta}\left|K_{\delta}^{\circ}\right|\right) \geq\left(\sum_{\delta}\left|P_{\delta}\right|\right)\left(\sum_{\delta}\left|Q_{\delta}\right|\right) \geq\left(\sum_{\delta} \sqrt{\left|P_{\delta}\right|\left|Q_{\delta}\right|}\right)^{2}
$$

where the sum is taken over all possible choices of $n$ signs $\delta$ and $K_{\delta}$ is a subset of $K$ corresponding to $\delta$. Finally,

$$
\begin{aligned}
|K|\left|K^{\circ}\right| & \geq\left(\sqrt{4^{-n}\left|B_{\infty}^{n}\right|\left|B_{1}^{n}\right|\left(1+c_{n}(1-t)\right)}+\left(2^{n}-1\right) \sqrt{4^{-n}\left|B_{\infty}^{n}\right|\left|B_{1}^{n}\right|}\right)^{2} \\
& =\left|B_{\infty}^{n}\right|\left|B_{1}^{n}\right|\left[1+2^{-n}\left(\sqrt{1+c_{n}(1-t)}-1\right)\right]^{2} \\
& \geq\left|B_{\infty}^{n}\right|\left|B_{1}^{n}\right|\left(1+2^{-n-1} c_{n}(1-t)\right) .
\end{aligned}
$$

Next we would like to review some properties and definitions about $\ell_{1}$ and $\ell_{\infty}$ sums, Hanner polytopes and their connections to graphs. We refer to [20,37] for more details.

Let $A, B$ be convex subsets of $\mathbb{R}^{n}$. The $\ell_{1}$-sum of $A$ and $B$ is defined by $\operatorname{conv}(A \cup$ $B$ ), the convex hull of the set $A \cup B$, and the $\ell_{\infty}$-sum is defined by $A+B$, the Minkowski sum of $A$ and $B$.

We recall that every Hanner polytope in $\mathbb{R}^{n}$ can be obtained from $n$ symmetric intervals in $\mathbb{R}^{n}$ by forming the $\ell_{1}$ or $\ell_{\infty}$ sums. In particular, a Hanner polytope in $\mathbb{R}^{n}$ is called standard if it is obtained from the intervals $\left[-e_{1}, e_{1}\right], \ldots,\left[-e_{n}, e_{n}\right]$. It is easy to see that every Hanner polytope is a linear image of a standard Hanner polytope. Moreover, each coordinate of any vertex of a standard Hanner polytope is 0 or \pm 1 .

The definition of a dual 0-1 polytope was given in 37] (the term "a dual 0-1 space" is used there, as a normed space whose unit ball is a dual 0-1 polytope): an unconditional polytope $P$ in $\mathbb{R}^{n}$ is called a dual 0-1 polytope if each coordinate of any vertex of $P$ and $P^{\circ}$ is 0 or \pm 1 . Every dual $0-1$ polytope $P$ in $\mathbb{R}^{n}$ can be associated with the graph $G=G(P)$ with the vertex set $\{1, \cdots, n\}$ and the edge set defined as follows:

$$
i, j \in\{1, \ldots, n\} \text { are connected by an edge of } G \text { if } e_{i}+e_{j} \notin P \text {. }
$$

We note that for each $i, j \in\{1, \ldots, n\}$ and for any dual 0 -1-polytope $P$ both the section and the orthogonal projection of $P$ by two-dimensional subspace spanned by $e_{i}$ and $e_{j}$ are dual 0-1 polytopes, that is, 2 -dimensional $\ell_{1}$ - or $\ell_{\infty}$-balls. It gives that $e_{i}+e_{j} \notin H$ if and only if $e_{i}+e_{j} \in H^{\circ}$, and thus $G\left(P^{\circ}\right)=\overline{G(P)}$ where $\bar{G}$ denotes the complement of $G$.

In addition, it turned out (see Theorem 2.5, 37]) that dual 0-1 polytopes in $\mathbb{R}^{n}$ are in one-to-one correspondence with perfect graphs on $\{1, \ldots, n\}$. In particular, as a special family of dual 0-1 polytopes, the standard Hanner polytopes in $\mathbb{R}^{n}$ are in one-to-one correspondence with the graphs on $\{1, \ldots, n\}$ which do not contain any induced path of edge length 3 (see [41] or Lemma 3.5 in [37]). Here, an induced path of a graph $G$ means a sequence of different vertices of $G$ such that each two adjacent vertices in the sequence are connected by an edge of $G$, and each two nonadjacent vertices in the sequence are not connected. Let $G$ be the graph associated with a 
dual 0-1 polytope $P$ as above. It turns out (Lemma 2.5, 37]) that a point $v \in \mathbb{R}^{n}$ is a vertex of $P$ if and only if each coordinate of $v$ is $-1,0$, or 1 , and the support

$$
\operatorname{supp}(v)=\left\{j:\left\langle v, e_{j}\right\rangle \neq 0\right\}
$$

of $v$ is a maximal independent set of $G$, i.e., a maximal set with respect to the set-inclusion such that no pair of elements is connected by an edge.

Note that if $K$ is unconditional, then we may apply a diagonal transformation to $K$ to get $B_{1}^{n} \subset K \subset B_{\infty}^{n}$. Indeed, this follows immediately from the fact that a tangent hyperplane with a normal vector $e_{i}$ touches $K$ at point $y_{i}$ which is a dilate of $e_{i}$. Thus to prove Theorem 1 it is enough to prove the following statement:

Equivalent form of Theorem 1 ; Let $K$ be an unconditional convex body in $\mathbb{R}^{n}$ satisfying $B_{1}^{n} \subset K \subset B_{\infty}^{n}$. If $\mathcal{P}(K) \leq(1+\varepsilon) \mathcal{P}\left(B_{\infty}^{n}\right)$ for some $\varepsilon>0$, then there exists a standard Hanner polytope $H$ in $\mathbb{R}^{n}$ such that $\mathrm{d}_{\mathcal{H}}(K, H)=O(\varepsilon)$.

Here $\mathrm{d}_{\mathcal{H}}$ is the Haussdorff distance $\mathrm{d}_{\mathcal{H}}$ of two sets $K, L \subset \mathbb{R}^{n}$ defined by

$$
\mathrm{d}_{\mathcal{H}}(K, L)=\max \left(\max _{x \in K} \min _{y \in L}|x-y|, \max _{y \in L} \min _{x \in K}|x-y|\right) .
$$

Proof of Theorem 1. We use induction on the dimension $n$ to prove the above statement. It is trivial if $n=1$, and the case $n=2$ was proved in [7]. Assume that the statement is true for all unconditional convex bodies of dimension $(n-1), n \geq 3$. For the inductive step, consider an unconditional convex body $K \subset \mathbb{R}^{n}$ satisfying

$$
B_{1}^{n} \subset K \subset B_{\infty}^{n} \quad \text { and } \quad \mathcal{P}(K) \leq(1+\varepsilon) \mathcal{P}\left(B_{\infty}^{n}\right) .
$$

We apply Lemma 1 and the inductive hypothesis to get standard Hanner polytopes $H_{1} \subset \mathbb{R}^{n} \cap e_{1}^{\perp}, \ldots, H_{n} \subset \mathbb{R}^{n} \cap e_{n}^{\perp}$ such that

$$
\mathrm{d}_{\mathcal{H}}\left(H_{j}, K \cap e_{j}^{\perp}\right)=O(\varepsilon), \quad \text { for } j=1, \ldots, n .
$$

Now we would like to show that we can "glue" these $(n-1)$-dimensional Hanner polytopes to create an $n$-dimensional Hanner polytope which is $O(\varepsilon)$-close to $K$. For each $j$, let $G_{j}=G\left(H_{j}\right)$ be the graph associated with $H_{j}$. Note that $\{1, \ldots, n\} \backslash\{j\}$ is the vertex set of $G_{j}$ and that $G_{j}$ does not contain an induced path of edge-length 3 .

Consider the graph $G$ with vertex set $\{1, \ldots, n\}$ and containing all edges of $G_{1}, \ldots, G_{n}$. Our goal is to show that the polytope $P$ corresponding to $G$ is a Hanner polytope which is $O(\varepsilon)$ - close to $K$.

We claim that the graph $G$ satisfies the following three properties.

1. Each $G_{j}$ is an induced subgraph of $G$ (i.e. for any $k, l \in\{1, \ldots, n\}, k$ and $l$ are connected by an edge of $G_{j}$ if and only if they are connected by an edge of $G$ ).

2. If $G$ is not the path of edge-length 3 , then $G$ does not contain any path of edge-length 3 as an induced subgraph.

If $G$ is the path of edge-length 3, then it is a perfect graph. Otherwise, the second property implies that $G$ does not contain any path of edge-length 3, that is, $G$ is the graph associated with a Hanner polytope. Thus, in any cases $G$ must be a perfect graph.

Let $P$ be the $0-1$ polytope associated with $G$, i.e., $G=G(P)$.

3. If $G$ is neither the complete graph nor its complement, then $\mathrm{d}_{\mathcal{H}}(K, P)=$ $O(\varepsilon)$. 
Indeed, to show the first property consider different $i, j, k \in\{1, \ldots, n\}$. Note that the existence of the $i j$ edge depends only on the properties of $K \cap \operatorname{span}\left\{e_{i}, e_{j}\right\}$, so $i, j$ are connected by an edge of $G_{k}$ if and only if they are connected by an edge of $G$. It implies that each $G_{k}$ is an induced subgraph of $G$.

To prove the second property, assume that $G$ is not the path of edge-length 3 . If $G$ contains a path of edge-length 3 , then there is $j \in\{1, \ldots, n\}$ which does not belong to this path. Thus, $G_{j}$, as an induced subgraph of $G$, must also contain the path of edge-length 3 , which contradicts with the definition of $G_{j}$.

To show the third property, we first note that if $G$ is not the complete graph, then every maximal independent set of $G$ is a proper subset of $\{1, \ldots, n\}$. The vertices of $P$ have coordinates 0,1 or -1 and the support of a vertex of $P$ is a maximal independent set of $G$. This implies that every vertex of $P$ has at least one zero coordinate, i.e. every vertex of $P$ is contained in one of coordinate hyperplanes. This, together with the inductive hypothesis, gives that each vertex of $P$ is $O(\varepsilon)$ close to the boundary of $K$, which means that $P$ is contained in $O(\varepsilon)$-neighborhood of $K$. It gives $K \supset(1+O(\varepsilon)) P$. Repeating the same argument for $\bar{G}$ we get $K^{\circ} \supset(1+O(\varepsilon)) P^{\circ}$ and thus $\mathrm{d}_{\mathcal{H}}(K, P)=O(\varepsilon)$.

Using the above three properties, we finish the proof of Theorem 1 modulo two pathological cases:

I. $G$ or $\bar{G}$ is the complete graph.

II. $G$ is the path of edge-length 3 .

Therefore, it remains to consider those cases:

Case I $(G$ (respectively $\bar{G})$ is the complete graph, so all $H_{1}, \ldots, H_{n}$ are the $(n-1)$ dimensional cubes (respectively $(n-1)$-dimensional cross-polytopes)). Taking the polar if necessary, we may assume, without loss of generality, that all $H_{1}, \ldots, H_{n}$ are the $(n-1)$-dimensional cubes. Let $\tilde{K}=\left(\frac{1}{1-\delta} K\right) \cap B_{\infty}^{n}$, where

$$
\delta=\min \left\{d:(1-d) H_{j} \subset K \cap e_{j}^{\perp}, \forall j=1, \ldots, n\right\} .
$$

Notice that $\delta=O(\varepsilon)$ and hence $\mathrm{d}_{\mathcal{H}}(K, \tilde{K})=O(\varepsilon)$. By (linear order) continuity of the volume product in the Hausdorff metric, we get $\mathcal{P}(\tilde{K}) \leq(1+O(\varepsilon)) \mathcal{P}(K)$. Thus, the assumption $\mathcal{P}(K) \leq(1+\varepsilon) \mathcal{P}\left(B_{\infty}^{n}\right)$ gives

$$
\mathcal{P}(\tilde{K}) \leq(1+O(\varepsilon)) \mathcal{P}\left(B_{\infty}^{n}\right) .
$$

Also, using $H_{j}=B_{\infty}^{n} \cap e_{j}^{\perp}$ and the definition of $\delta$ we get

$$
\tilde{K} \cap e_{j}^{\perp}=B_{\infty}^{n} \cap e_{j}^{\perp}, \quad \forall j=1, \ldots, n .
$$

Consider $p=(t, \ldots, t) \in \partial \tilde{K}$. Then Lemma 2 gives

$$
\mathcal{P}(\tilde{K}) \geq(1+c(1-t)) \mathcal{P}\left(B_{\infty}^{n}\right) .
$$

Together with (12) the above inequality implies that $1-t=O(\varepsilon)$. Thus $p$ is in $O(\varepsilon)$-neighborhood of the vertex $(1, \ldots, 1)$ of $B_{\infty}^{n}$. The unconditionality of $\tilde{K}$ and (13) give that $\mathrm{d}_{\mathcal{H}}\left(\tilde{K}, B_{\infty}^{n}\right)=O(\varepsilon)$ and hence $\mathrm{d}_{\mathcal{H}}\left(K, B_{\infty}^{n}\right)=O(\varepsilon)$.

Case II ( $G$ is the path of edge-length 3 ). The direct computation below will show that this case contradicts with the assumption on the volume product of $K$. Indeed in this case $K \subset \mathbb{R}^{4}$ is $O(\varepsilon)$-close to the polytope $P$ which is associated with the path of edge-length 3 . The volume product of $P$ is strictly greater than that of the cube $B_{\infty}^{4}$. Thus selecting $\varepsilon$ small enough we will be able to contradict the 
assumption of Theorem 1. The details of calculation may be found in [27, 36]; for the sake of completeness we also present them here. Let $G$ be represented by the vertex set $\{1,2,3,4\}$ and the edge set $\{12,23,34\}$; then $\bar{G}$ has the same vertex set and the edge set $\{24,41,13\}$. Applying the characterization of vertices of a polytope by maximal independent sets, we can get

$$
P=\operatorname{conv}\{( \pm 1,0, \pm 1,0),(0, \pm 1,0, \pm 1)\}
$$

and

$$
P^{\circ}=\operatorname{conv}\{( \pm 1, \pm 1,0,0),(0,0, \pm 1, \pm 1)\} .
$$

Then we have $|P|=\left|P^{\circ}\right|$ and

$$
\begin{aligned}
|P| & =|\operatorname{conv}\{( \pm 1,0, \pm 1,0),(0, \pm 1,0, \pm 1)\}| \\
& =\left|\left\{x \in \mathbb{R}^{4}:\left|x_{1}\right|+\left|x_{2}\right| \leq 1,\left|x_{2}\right|+\left|x_{3}\right| \leq 1,\left|x_{3}\right|+\left|x_{4}\right| \leq 1\right\}\right| \\
& =2 \int_{0}^{1}\left|\left\{x \in \mathbb{R}^{3}:\left|x_{1}\right|+\left|x_{2}\right| \leq 1,\left|x_{2}\right|+\left|x_{3}\right| \leq 1,\left|x_{3}\right| \leq 1-s\right\}\right| d s \\
& =4 \int_{0}^{1} \int_{0}^{1-s}\left|\left\{x \in \mathbb{R}^{3}:\left|x_{1}\right|+\left|x_{2}\right| \leq 1,\left|x_{2}\right| \leq 1-t\right\}\right| d t d s \\
& =8 \int_{0}^{1} \int_{0}^{1-s}\left(1-t^{2}\right) d t d s=\frac{10}{3} .
\end{aligned}
$$

Thus $\mathcal{P}(P)=\left(\frac{10}{3}\right)^{2}>\frac{4^{4}}{4 !}=\mathcal{P}\left(B_{\infty}^{4}\right)$. On the other hand, note that in this case $G$ and $\bar{G}$ are not complete graphs, so we may apply the Property 3 from above to claim that $K$ is $O(\varepsilon)$-close to $P$. Therefore, $\mathcal{P}(K)>\mathcal{P}\left(B_{\infty}^{4}\right)$, which contradicts the assumption $\mathcal{P}(K) \leq(1+\varepsilon) \mathcal{P}\left(B_{\infty}^{4}\right)$ for $\varepsilon>0$ small enough.

\section{Minimality near UnCOnditional CONVEX Bodies}

The following local minimality result, proved in 20, is the main tool of this section

Theorem 3. Let $K$ be a symmetric convex body in $\mathbb{R}^{n}$ close to one of Hanner polytopes in $\mathbb{R}^{n}$ in the sense that

$$
\min \left\{\mathrm{d}_{\mathcal{B} \mathcal{M}}(K, H): H \text { is a Hanner polytope in } \mathbb{R}^{n}\right\}=1+\varepsilon
$$

for $0<\varepsilon \leq \varepsilon(n)$. Then

$$
\mathcal{P}(K) \geq \mathcal{P}\left(B_{\infty}^{n}\right)+c(n) \varepsilon
$$

where $c(n)>0$ is a constant depending on the dimension $n$ only.

Let $\gamma_{n}>0$ be a common threshold of $\varepsilon$ to satisfy both Theorem 1 and Theorem 3. More precisely, the constant $\gamma_{n}$ satisfies the following:

If $K$ is a symmetric convex body in $\mathbb{R}^{n}$ with $\mathrm{d}_{\mathcal{B M}}(K, H)=1+\varepsilon, \varepsilon \leq \gamma_{n}$, for some Hanner polytope $H$, then

$$
\mathcal{P}(K) \geq\left(1+\alpha_{n} \varepsilon\right) \mathcal{P}\left(B_{\infty}^{n}\right) .
$$


If $K$ is an unconditional convex body in $\mathbb{R}^{n}$ with $\mathrm{d}_{\mathcal{B M}}(K, H) \geq 1+\varepsilon$, $\varepsilon \leq \gamma_{n}$, for every Hanner polytope $H$, then

$$
\mathcal{P}(K) \geq\left(1+\beta_{n} \varepsilon\right) \mathcal{P}\left(B_{\infty}^{n}\right) .
$$

where $\alpha_{n}, \beta_{n}>0$ are constants depending on $n$ only.

Proof of Theorem 2. We will use $\gamma_{n}$ to select the constant $\varepsilon_{n}$, a threshold of $\varepsilon$ in the statement of the Theorem 2. Consider a convex symmetric body $K \subset \mathbb{R}^{n}$. Let $L \subset \mathbb{R}^{n}$ be an unconditional convex body with the smallest, among unconditional bodies, Banach-Mazur distance to $K$ and let $\varepsilon \geq 0$ be such that $\mathrm{d}_{\mathcal{B M}}(K, L)=1+\varepsilon$. Also consider a Hanner polytope $H_{0}$ with the smallest, among Hanner polytopes, Banach-Mazur distance to $L$ and let $\delta \geq 0$ be such that $\mathrm{d}_{\mathcal{B M}}\left(L, H_{0}\right)=1+\delta$.

We will consider two cases: $\delta \leq \gamma_{n} / 3$ and $\delta>\gamma_{n} / 3$.

1. Assume $\delta \leq \frac{\gamma_{n}}{3}$ and thus $\mathrm{d}_{\mathcal{B} \mathcal{M}}\left(L, H_{0}\right) \leq 1+\frac{\gamma_{n}}{3}$. Then

$$
\begin{aligned}
\mathrm{d}_{\mathcal{B M}}\left(K, H_{0}\right) & \leq \mathrm{d}_{\mathcal{B M}}(K, L) \mathrm{d}_{\mathcal{B M}}\left(L, H_{0}\right)=(1+\varepsilon)(1+\delta) \\
& \leq 1+\varepsilon+(1+\varepsilon) \gamma_{n} / 3 \leq 1+\gamma_{n} / 3+2 \gamma_{n} / 3=1+\gamma_{n},
\end{aligned}
$$

where, to guarantee the above inequalities, we select $\varepsilon_{n} \leq \min \left\{\frac{\gamma_{n}}{3}, 1\right\}$. Thus, by (14),

$$
\mathcal{P}(K) \geq\left(1+\alpha_{n} \varepsilon\right) \mathcal{P}\left(B_{\infty}^{n}\right) .
$$

2. Now assume that $\delta>\frac{\gamma_{n}}{3}$ and thus $\mathrm{d}_{\mathcal{B} \mathcal{M}}(L, H)>1+\frac{\gamma_{n}}{3}$ for every Hanner polytope $H$. We will require $\varepsilon_{n} \leq \min \left\{\frac{\beta_{n} \gamma_{n}}{12 n}, \frac{1}{2 n}\right\}$. Since $L \subset T K \subset$ $(1+\varepsilon) L$ for some $T \in \mathrm{GL}(n)$,

$$
\begin{aligned}
\mathcal{P}(K) & =\mathcal{P}(T K) \geq|L|\left|(1+\varepsilon)^{-1} L^{\circ}\right|=(1+\varepsilon)^{-n} \mathcal{P}(L) \\
& \geq(1-\varepsilon)^{n} \mathcal{P}(L) \geq(1-n \varepsilon) \mathcal{P}(L) .
\end{aligned}
$$

Moreover, since $\mathcal{P}(L) \geq\left(1+\beta_{n} \gamma_{n} / 3\right) \mathcal{P}\left(B_{\infty}^{n}\right)$ by (15),

$$
\begin{aligned}
\mathcal{P}(K) & \geq(1-n \varepsilon) \mathcal{P}(L) \geq(1-n \varepsilon)\left(1+\beta_{n} \gamma_{n} / 3\right) \mathcal{P}\left(B_{\infty}^{n}\right) \\
& \geq(1-n \varepsilon)(1+4 n \varepsilon) \mathcal{P}\left(B_{\infty}^{n}\right)=\left(1+3 n \varepsilon-4 n^{2} \varepsilon^{2}\right) \mathcal{P}\left(B_{\infty}^{n}\right) \\
& \geq(1+n \varepsilon) \mathcal{P}\left(B_{\infty}^{n}\right) .
\end{aligned}
$$

Finally we combine the above two cases by taking

$$
\begin{aligned}
& \varepsilon_{n}=\min \left\{\frac{\gamma_{n}}{3}, \frac{\beta_{n} \gamma_{n}}{12 n}, \frac{1}{2 n}\right\} \\
& \tau_{n}=\min \left\{\alpha_{n}, n\right\} .
\end{aligned}
$$

Then, $\mathcal{P}(K) \geq\left(1+\tau_{n} \varepsilon\right) \mathcal{P}\left(B_{\infty}^{n}\right)$ whenever $\varepsilon \leq \varepsilon_{n}$.

\section{REFERENCES}

[1] S. Artstein-Avidan, B. Klartag, and V. Milman, The Santaló point of a function, and a functional form of the Santaló inequality, Mathematika 51 (2004), no. 1-2, 33-48 (2005), DOI 10.1112/S0025579300015497. MR2220210(2007a:52008)

[2] Keith Ball, Logarithmically concave functions and sections of convex sets in $\mathbf{R}^{n}$, Studia Math. 88 (1988), no. 1, 69-84. MR932007(89e:52002)

[3] F. Barthe, K.J. Böröczky, and M. Fradelizi, Stability of the functional forms of the BlaschkeSantaló inequality, Monatsh. Math. 173 (2014), no 2, 135-159/

[4] F. Barthe and M. Fradelizi, The volume product of convex bodies with many hyperplane symmetries, Amer. J. Math. 135 (2013), no. 2, 311-347, DOI 10.1353/ajm.2013.0018. MR 3038713 
[5] Károly J. Böröczky, Stability of the Blaschke-Santaló and the affine isoperimetric inequality, Adv. Math. 225 (2010), no. 4, 1914-1928, DOI 10.1016/j.aim.2010.04.014. MR2680195 (2011j:52016)

[6] Károly J. Böröczky and Daniel Hug, Stability of the reverse Blaschke-Santaló inequality for zonoids and applications, Adv. in Appl. Math. 44 (2010), no. 4, 309-328, DOI 10.1016/j.aam.2009.09.002. MR2600783 (2011c:52005)

[7] K.J. Böröczky, E. Makai, M. Meyer, and S. Reisner, On the volume product of planar polar convex bodies. Lower estimates with stability, Studia Sci. Math. Hungar. 50 (2013), 159-198.

[8] J. Bourgain and V. D. Milman, New volume ratio properties for convex symmetric bodies in $\mathbf{R}^{n}$, Invent. Math. 88 (1987), no. 2, 319-340, DOI 10.1007/BF01388911. MR.880954 (88f:52013)

[9] M. Fradelizi, Y. Gordon, M. Meyer, and S. Reisner, The case of equality for an inverse Santaló functional inequality, Adv. Geom. 10 (2010), no. 4, 621-630, DOI 10.1515/ADVGEOM.2010.026. MR2733956 (2011j:52017)

[10] Matthieu Fradelizi and Mathieu Meyer, Increasing functions and inverse Santaló inequality for unconditional functions, Positivity 12 (2008), no. 3, 407-420, DOI 10.1007/s11117-0072145-z. MR2421143(2009e:26025)

[11] M. Fradelizi and M. Meyer, Some functional inverse Santaló inequalities, Adv. Math. 218 (2008), no. 5, 1430-1452, DOI 10.1016/j.aim.2008.03.013. MR2419928(2009f:26026)

[12] M. Fradelizi and M. Meyer, Functional inequalities related to Mahler's conjecture, Monatsh. Math. 159 (2010), no. 1-2, 13-25, DOI 10.1007/s00605-008-0064-0. MR2564385 (2011a:39041)

[13] Matthieu Fradelizi, Mathieu Meyer, and Artem Zvavitch, An application of shadow systems to Mahler's conjecture, Discrete Comput. Geom. 48 (2012), no. 3, 721-734, DOI 10.1007/s00454012-9435-3. MR2957641

[14] A. Giannopoulos, G. Paouris, and B. Vritsiou, The isotropic position and the reverse Santalo inequality, to appear in Israel J. Math.

[15] Y. Gordon and M. Meyer, On the Minima of the Functional Mahler Product, Houston J. Math. 40 (2014), no. 2, 385-393.

[16] Y. Gordon, M. Meyer, and S. Reisner, Zonoids with minimal volume-product-a new proof, Proc. Amer. Math. Soc. 104 (1988), no. 1, 273-276, DOI 10.2307/2047501. MR958082 (89i:52015)

[17] Olof Hanner, Intersections of translates of convex bodies, Math. Scand. 4 (1956), 65-87. MR0082696 (18,595b)

[18] Allan B. Hansen and Asvald Lima, The structure of finite-dimensional Banach spaces with the 3.2. intersection property, Acta Math. 146 (1981), no. 1-2, 1-23, DOI 10.1007/BF02392457. MR.594626 (82g:46037)

[19] Fritz John, Extremum problems with inequalities as subsidiary conditions, Studies and Essays Presented to R. Courant on his 60th Birthday, January 8, 1948, Interscience Publishers, Inc., New York, N. Y., 1948, pp. 187-204. MR0030135(10,719b)

[20] J. Kim, Minimal volume product near Hanner polytopes, J. Funct. Anal., 266 (2014), no. 4, 2360-2402.

[21] Jaegil Kim and Han Ju Lee, Strong peak points and strongly norm attaining points with applications to denseness and polynomial numerical indices, J. Funct. Anal. 257 (2009), no. 4, 931-947, DOI 10.1016/j.jfa.2008.11.024. MR2535458(2010m:46069)

[22] Jaegil Kim and Shlomo Reisner, Local minimality of the volume-product at the simplex, Mathematika 57 (2011), no. 1, 121-134, DOI 10.1112/S0025579310001555. MR2764160 (2012a:52023)

[23] Greg Kuperberg, From the Mahler conjecture to Gauss linking integrals, Geom. Funct. Anal. 18 (2008), no. 3, 870-892, DOI 10.1007/s00039-008-0669-4. MR2438998(2009i:52005)

[24] K. Mahler, Ein Minimalproblem für konvexe Polygone, Mathematica (Zutphen) B7 (1939), 118-127.

[25] Kurt Mahler, Ein Übertragungsprinzip für konvexe Körper (German), Časopis Pěst. Mat. Fys. 68 (1939), 93-102. MR0001242 (1,202c)

[26] E. Makai, Jr., The recent status of the volume product problem, to appear in Banach Center Publications. Also available from http://www.renyi.hu/ makai/jurata.pdf 
[27] Mathieu Meyer, Une caractérisation volumique de certains espaces normés de dimension finie (French, with English summary), Israel J. Math. 55 (1986), no. 3, 317-326, DOI 10.1007/BF02765029. MR876398 (88f:52017)

[28] Mathieu Meyer, Convex bodies with minimal volume product in $\mathbf{R}^{2}$, Monatsh. Math. 112 (1991), no. 4, 297-301, DOI 10.1007/BF01351770. MR.1141097 (92k:52015)

[29] Mathieu Meyer and Alain Pajor, On Santaló's inequality, Geometric aspects of functional analysis (1987-88), Lecture Notes in Math., vol. 1376, Springer, Berlin, 1989, pp. 261-263, DOI 10.1007/BFb0090059. MR.1008727 (90h:52012)

[30] M. Meyer and S. Reisner, Inequalities involving integrals of polar-conjugate concave functions, Monatsh. Math. 125 (1998), no. 3, 219-227, DOI 10.1007/BF01317315. MR1614279 (99c:52004)

[31] Mathieu Meyer and Shlomo Reisner, Shadow systems and volumes of polar convex bodies, Mathematika 53 (2006), no. 1, 129-148 (2007), DOI 10.1112/S0025579300000061. MR2304056(2008d:52010)

[32] Fedor Nazarov, The Hörmander proof of the Bourgain-Milman theorem, Geometric aspects of functional analysis, Lecture Notes in Math., vol. 2050, Springer, Heidelberg, 2012, pp. 335343, DOI 10.1007/978-3-642-29849-3_20. MR2985302

[33] Fedor Nazarov, Fedor Petrov, Dmitry Ryabogin, and Artem Zvavitch, A remark on the Mahler conjecture: local minimality of the unit cube, Duke Math. J. 154 (2010), no. 3, 419430, DOI 10.1215/00127094-2010-042. MR2730574(2012a:52010)

[34] C. M. Petty, Affine isoperimetric problems, Discrete geometry and convexity (New York, 1982), Ann. New York Acad. Sci., vol. 440, New York Acad. Sci., New York, 1985, pp. 113127, DOI 10.1111/j.1749-6632.1985.tb14545.x. MR809198(87a:52014)

[35] Shlomo Reisner, Zonoids with minimal volume-product, Math. Z. 192 (1986), no. 3, 339-346, DOI 10.1007/BF01164009. MR845207 (87g:52022)

[36] Shlomo Reisner, Minimal volume-product in Banach spaces with a 1-unconditional basis, J. London Math. Soc. (2) 36 (1987), no. 1, 126-136, DOI 10.1112/jlms/s2-36.1.126. MR897680 (88h:46029)

[37] S. Reisner, Certain Banach spaces associated with graphs and CL-spaces with 1-unconditional bases, J. London Math. Soc. (2) 43 (1991), no. 1, 137-148, DOI 10.1112/jlms/s2-43.1.137. MR:1099093 (92e:52004)

[38] Shlomo Reisner, Carsten Schütt, and Elisabeth M. Werner, Mahler's conjecture and curvature, Int. Math. Res. Not. IMRN 1 (2012), 1-16. MR2874925

[39] D. Ryabogin and A. Zvavitch, Analytic methods in convex geometry in Analytical and Probabilistic Methods in the Geometry of Convex Bodies, IMPAN Lecture Notes, vol. 2, Warsaw (2014), 87-183.

[40] L. A. Santaló, Un invariante afin para los cuerpos convexos del espacio de $n$ dimensiones, Portugal. Math. 8 (1949), 155-161.

[41] D. Seinsche, On a property of the class of $n$-colorable graphs, J. Combinatorial Theory Ser. B 16 (1974), 191-193. MR0337679 (49 \#2448)

[42] Rolf Schneider, Convex bodies: the Brunn-Minkowski theory, Encyclopedia of Mathematics and its Applications, vol. 44, Cambridge University Press, Cambridge, 1993. MR.1216521 (94d:52007)

[43] J. Saint-Raymond, Sur le volume des corps convexes symétriques (French), Initiation Seminar on Analysis: G. Choquet-M. Rogalski-J. Saint-Raymond, 20th Year: 1980/1981, Publ. Math. Univ. Pierre et Marie Curie, vol. 46, Univ. Paris VI, Paris, 1981, pp. Exp. No. 11, 25. MR670798(84j:46033)

[44] Alina Stancu, Two volume product inequalities and their applications, Canad. Math. Bull. 52 (2009), no. 3, 464-472, DOI 10.4153/CMB-2009-049-0. MR2547812 (2011b:52011)

[45] Terence Tao, Structure and randomness, American Mathematical Society, Providence, RI, 2008. Pages from year one of a mathematical blog. MR 2459552 (2010h:00002)

[46] T. Tao, Santalo inequalities, http://www.math.ucla.edu/ tao/preprints/Expository/ santalo.dvi 
Department of Mathematical and Statistical Sciences, University of Alberta, Edmonton, Alberta, T6G 2G1, Canada

E-mail address: jaegil@ualberta.ca

Department of Mathematical Sciences, Kent State University, Kent, Ohio 44242

E-mail address: zvavitch@math.kent.edu 